\title{
ISLAMOPHOBIA AND RELIGIOUS INTOLERANCE: THREATS TO GLOBAL PEACE AND HARMONIOUS CO-EXISTENCE
}

\author{
Kazeem Oluwaseun DAUDA \\ National Open University of Nigeria (NOUN), Jabi-Abuja, Nigeria \\ Consultant, FARKAZ Technologies \& Education Consulting Int'l, Ijebu-Ode \\ seundelaw@gmail.com
}

\begin{abstract}
Recent events show that there are heightened fear, hostilities, prejudices and discriminations associated with religion in virtually every part of the world. It becomes almost impossible to watch news daily without scenes of religious intolerance and violence with dire consequences for societal peace. This paper examines the trends, causes and implications of Islamophobia and religious intolerance for global peace and harmonious co-existence. It relies on content analysis of secondary sources of data. It notes that fear and hatred associated with Islām and persecution of Muslims is the fallout of religious intolerance as reflected in most melee and verbal attacks, anti-Muslim hatred, racism, xenophobia, anti-Sharī'ah policies, high-profile terrorist attacks, and growing trends of far-right or right-wing extremists. It reveals that Islamophobia and religious intolerance have led to proliferation of attacks on Muslims, incessant loss of lives, wanton destruction of property, violation of Muslims' fundamental rights and freedom, rising fear of insecurity, and distrust between Muslims and
\end{abstract}


non-Muslims. The paper concludes that escalating Islamophobic attacks and religious intolerance globally had constituted a serious threat to world peace and harmonious co-existence. Relevant resolutions in curbing rising trends of Islamophobia and religious intolerance are suggested.

Keywords: Global peace, harmonious co-existence, Islamophobia, religious intolerance.

\section{A. Introduction}

Religion remains one of the significant sources of intolerance, discrimination and marginalisation across the globe. In contemporary times, the activities of religions rather than encouraging peace and mutual tolerance, have exacerbated fear, mistrust and negative feelings, among their respective followers. Peaceful co-existence in the world has often been jeopardised and threatened by refusal of one religious or non-religious group to tolerate beliefs and practices of other persons based on their religious affiliations. Shocking and breaking news on daily basis reflects violence related to religion. As Limon, Ghanea and Power (2014:2) has observed, "it is almost impossible to turn on the news today without witnessing scenes of hatred, violence and intolerance perpetrated in the name of religion or belief." This shows that there are increasingly religious hostilities and discriminations in virtually every part of the world.

Islām teaches that we live in peace and harmony with people of different faiths, including Christians, Jews and others. Islām is a religion of peace - in theory, principles and praxis - that is against any form of oppression, discrimination, 
stereotypes, persecution and unjust killing of innocent souls. However, there had been a massive surge of hatred against Islām and its adherents in recent past. Over the years, especially since the post-9/11 U.S. attacks, Islamophobia has not only been on the rise in the Americas, Australia, Europe, Asia and Africa, it has reached an intractable point and growing strongly in these continents (OIC Observatory Report, 2018).

Consequently, Muslims have become targets of abusive and violent attacks. Islamophobic violence against Muslims has taken different patterns, including, physical assaults, verbal abuse, mass killing, bombing and destruction of Mosques, Islamic centres, schools, attires and Islām's sacred symbols, as well as Muslim tombs and communities (Abu Sway, 2005; Runnymede Trust, 1997). For instance, forty-five attacks were recorded against Muslims, persons mistaken for Muslims and Mosques in the United States in 2015 alone, while in 2017, five different Mosques in Texas, Washington, Florida and Michigan, caught aflame (Yahoo News cited in Ghani, 2019: 7).

Paradoxically, the tragic rise in hostility towards Islamic faith and Muslims in the world is largely reflected in the wide scale of negative narratives of Islām in the mainstream media, literature, and public discourses. Thus, the intensity with which Islamophobia and religious intolerance are spreading poses a serious concern that requires continuous interrogation in a bid to contain the menaces. Meanwhile, extant studies have focused on the review of Islamophobic discourse or causes and effect of Islamophobia but no scholarly writing to the author's knowledge is devoted to interrogate the perceived threats of Islamophobia and religious intolerance to peaceful 
co-existence the world over. Therefore, the central focus of this paper is to examine Islamophobia and religious intolerance with particular emphasis on their causes and implications for societal peace and harmonious co-existence around the globe.

\section{B. Discussion}

\section{Islamophobia and religious intolerance: a theoretical and conceptual analysis}

Scholars differ in their conceptual understanding on the term "Islamophobia". Suffice to note that the prominent use of the concept began with the report of the Runnymede Trust's Commission on British Muslims and Islamophobia (CBMI) entitled Islamophobia: a Challenge for Us All (1997). The Trust provided a foundation upon which many other definitions emerged. According to the Commission, Islamophobia is "unfounded hostility towards Islām and practical consequences of such hostility in unfair discrimination against Muslim individuals and communities" (Runnymede Trust, 1997: 4). This definition reflects that Islamophobia could occur at both the individual and group levels. In a similar trend, Kumar (2018) argues that daily acts of hostility, hate crimes, and even job discrimination against Muslims are the outward manifestations of Islamophobia.

Moreover, Islamophobia involves socially reproduced prejudices and aversion to Islām and Muslims, as well as actions and practices that attack, exclude or discriminate against persons on the basis that they are or perceived to be Muslim (Gardell, 2010). It is an institutionalised, structural, and systemic war constituting a systemic form of racism and discrimination against anyone who is perceived as associated 
with Islām and an intense hatred or fear of Islām, and hostility or prejudice towards Muslims (Abdulhadi, 2018: 14). Islamophobia also involves discrimination against Muslims in employment and in the provision of health services, exclusion from managerial positions and jobs of high responsibility, as well as discrimination and exclusion from political and governmental posts (Abu Sway, 2005; Runnymede Trust, 1997).

In a broader context, Abadi (2018: 5) synonymously used harsh talk, systemic and institutional form of racism, silencing of Muslim voices, selective condemnation, and constant embarrassment at airport, to explain Islamophobia. Abadi further describes Islamophobia as when travelling to U.S. from Muslims countries is banned, when the Muslim communities are placed under FBI's surveillance and policing, when extremist threat is measured by merely speaking Arabic, and when Muslims are made to apologise for crimes they did not commit. Abadi's conception captures the pervasive nature and endangering characteristics of contemporary Islamophobia in western countries, particularly the United States. It also suggests that Islamophobia is a new form of racism. This is buttressed by the Runnymede Trust (2017) in its $20^{\text {th }}$ Anniversary report which states that Islamophobia is anti-Muslim racism with the purpose of impairing the recognition, enjoyment or exercise of human rights, and fundamental freedoms of Muslims.

Flowing from the foregoing positions, Islamophobia in this context is a combination of two nuanced words: Islām - an Arabic word which signifies religion that exists to reflect total and voluntary submission to God Almighty, by those who desires peace, equity and wholeness, called Muslims; and phobia which 
means fear of something due to danger inherent in it. Thus, Islamophobia connotes uninformed fear associated with Islām by non-Muslims that do not believe in the peaceful notions of the religion of Islām. It is the term used to describe the hatred, negative stereotypes and hostility perpetrated toward Muslims due to wrong perception about Islām which reflects in verbal and physical attacks, harassment, discrimination, torture, murder, bombing, kidnapping, arson and vandalism, use of media agencies, hate speech, intimidation and marginalisation, and demonstrations, rallies and marches, as well as distribution of leaflets and other literature.

Religious intolerance is abroad concept. The relationship between religion and intolerance has been the subject of intense discourse. This is because religion and intolerance are inextricably linked. Religion as old as mankind has played a significant role in the human existence and interaction. This implies that religion is a universal phenomenon that largely revolves around the existence of man. Put differently, religion satisfies the spiritual and moderate material needs of man. It could mean the set of beliefs, feelings and practices defining the relations between human being and the supernatural. In other words, religion is man's efforts to establish and maintain cordial relations between them and spiritual powers on the one hand, and fellow man and other creatures on the other (Kareem, 2014). Etymologically, the term "religion" is derived from Latin word religare meaning "to create a tie to God" or "reunite with God." As a religionist, one could either belong to a polytheist, pantheist or monotheist.

Considering the trend of monotheistic religion across the world, one is convinced that what religion stands for is 
peace and tranquillity in different societies, but the ignorance and the excesses of the adherents had brought its negative impact on the global societies. Conversely, intolerance has replaced tolerance amidst violence. Intolerance refers to the unwillingness to recognise and accept differences in opinions or beliefs of others. It has to do with difficulty to bear or endure the beliefs that are different from one's own (https:// www.vocabulary.com/dictionary/intolerance).

The Encyclopedia of World Problems and Human Potential (2017) explains that religious intolerance includes acts of denying the right of people of another religious faith to practice and express their beliefs freely. It is a blind refusal to understand and respect views or positions that are opposed to one's cherished religious views. Raji, Abdullateef, Araba-Yusuf and Festus (2015) are of the view that religious intolerance involves when a particular group refuses to accept or accommodate the views, opinions of other opposing religious adherent, throw verbal insults at members of another religious group, attack with dangerous missiles, put up physical fights, deny some religious groups some fundamental social services, such as provision of land to build worship places, and mutilate any of the religious' holy books or tombs.

For the purpose of this study, therefore, religious intolerance implies the hostility, inability and difficulty in accepting other religious views, perhaps due to wrong religious ideology or bigotry. It is expressed in discrimination, repression and religious rivalry, and result in persecution. Religious intolerance leads to violence, distrust, disintegration, hostility and persistent hatred among religious groups within nations. 


\section{Historical context and emergence of Islamophobic discourse}

Apparently, the emergence of "Islamophobia" has received tremendous scholarly attention with emphasis on its historical context, as well as the interplay between western cultures and fear of Islām and Muslims. According to Vertovec (2002), Islamophobia has not necessarily escalated in the past decades, but there has been increased scrutiny of it in public and scholarly discourse. Reflecting on the structural character and history of Islamophobia, Abdulhadi (2018) emphasizes that "Islamophobia" is not a new phenomenon, its historical roots can be traced to the European Crusades in the 11th to 13th centuries and expulsion of Muslim Arabs and Africans from Andalucía in the late 15th century. He adds that Islamophobia is rooted in colonialism by the European powers, as well as Orientalism and East-West dichotomy.

Similarly, Bleich (2011) has traced the coinage of "Islamophobia" as a new word to the publication of Edward Said's (1978) classic work, entitled Orientalism in the late 1970s. In this context, it was established and widely agreed that "the West" has long associated Islām with negative images, sentiments, and stereotypes. Orientalism describes the way western cultural, academic, and imperial projects have crafted a dehumanising representation of "the Arab" as an "exotic and barbarous Orients". By decoding the body of writing that compares a "civilised" West to a "backwards" Arab world, Orientalism provides one of the earliest critiques of stigmatised Muslim identities and the way in which Orientalists exploited the negative stereotypes of Eastern cultures as a justification for colonial ambitions (Itaoui \& Elsheikh, 2018: 7). 
For Benn and Jawad (2003), Islamophobia has increased since Ayatollah Khomeini's 1989 fatwa which incited Muslims to attempt to murder Salman Rushdie, the author of The Satanic Verses, and since the 11 September 2001 attacks. In the same vein, the International Civil Liberties Alliance [ICLA] (2013) points out that "Islamophobia" was not recorded in English until the second half of the 20th century. ICLA notes further that its use became more widespread in the late 1980s, in the wake of the Iranian fatwa against Salman Rushdie over his book The Satanic Verses. Likewise, Hasan (2003 cited in ICLA, 2013) maintains that the term "Islamophobia" first appeared in Britain during the Rushdie affair in the late 1980's, in an attempt to silence critics, such as Rushdie and his supporters, for free speech by arguing that only the wider 'Islamophobia' of British society and states allowed this to go unpunished.

In the views of Istriyani and Yuliatun (2016), the study of Islamophobia began in 1995 in England, while some studies show that Islām is perceived as a threat, while others consider it to have similarities with the Nazi and Communism that contains an overview of invasion, domination and infiltration. Bleich (2011) insists that Islamophobia originally developed in the late 1990s to draw attention to harmful rhetoric and actions directed at Islām and Muslims in western countries. For Lee, Gibbons, Thompson and Timani (2009), and Zúquete (2008), "Islamophobia" emerged in contemporary discourse with the 1997 publication of the report "Islamophobia: a challenge for us all" by the Runnymede Trust (1997). This publication indicates that the term "Islamophobia" has been popularised because of the resurgence in the post-Cold War and post-9/11 phenomenon in United States. 
Esposito (2017: xiv) asserts that Islamophobia like antiSemitism and xenophobia, is historically rooted, but traced its contemporary resurgence to the significant influx of Muslims to the West in the late 20th century, the Iranian revolution, hijackings, hostage taking and other acts of terrorism in the 1980s and 1990s, as well as terrorist attacks on the U.S. World Trade Centre and the Pentagon in 2001 and subsequent terrorist attacks in Europe and other world countries. Since then, concept of Islamophobia has been regularly used by scholars, media outfits, public speakers, political leaders, and non-governmental organisations (NGOs) in Britain, France, and United States, among other countries. Notwithstanding the differences in the scholarly perceptions on the historical contextand emergence of Islamophobia, it is indeed imperative to point out that the year 2000s has signalled recurrence in Islamophobic incidents and hate crimes against the followers of Islām, their places of worship, and where other religious activities taking place.

\section{Panoramic view, cases and causes of Islamophobia and religious intolerance}

Across the globe, particularly in Canada, U.S., Australia, U.K., New Zealand, Poland, Hungary, Sweden, Norway, China, Myanmar, Sri Lanka, India, Vietnam, Palestine, Central African Republic (CAR), and a host of others, violent confrontations, anti-Islām rallies, hate crimes, mass killings and murder of Muslims, and bombing of Mosques and Muslim graveyards, induced by Islamophobia, is quite alarming. It is unassailable that Islamophobia has existed in premise before the 9/11 terrorist attacks but has increased in frequency and notoriety 
in the post-9/11 era (Gallup News, n.d.). According to Public Radio International (12 September 2016 cited in Edward, 2018), Islamophobia did not pose a common and constant threat to American Muslims until after 9/11 attacks. There is continued surge in anti-Muslim attacks in the United States. The country has recorded over 1,200 anti-Mosque incidents since 2005 (see American Civil Liberties Union, 2019).

Recent surveys conducted in Europe indicate a rise in Islamophobia since 2015 (Mohamed Osman, 2017). Mohamed Osman claims that series of onslaughts by Right-wing nationalist parties against Muslims have resulted in the rise of Islamophobia in Europe and North America, while the rise of the Hindu nationalist movement in India has seen the forced conversion of Muslims and increased incidences of violence against Muslims (Mohamed Osman, 2017). A large-scale poll published in 2011 revealed that 48.6 percent of Australians identify themselves as having anti-Muslim attitudes $(A B C$ News, 23 February 2011), while another survey published in 2014 showed that a quarter of Australians held negative attitudes toward Muslims than any other religious group (Chalkley-Rhoden, 2014).

Statistics Canada found that hate crimes against Muslims in Canada grew 253 percent from 2012 to 2015 (TheConversation, 6 February 2019). In the U.K., antiMuslim attacks and abuse in 2017 rose by $26 \%$ with 1,201 recorded incidents (Marsh, 2018). Furthermore, there were 813 attacks against Muslims and Mosques in year 2018 in Germany (TRTWorld Magazine, 16 May 2019). The terrorist attacks on two Mosques in Christchurch, New Zealand on 15 March 2019, left fifty-one Muslims dead and over fifty injured 
(Graham-McLay, 2019). In Australia, over seven incidents could be considered Islamophobic. Moreover, Muslims have been massacred in several terrorist and melee attacks related to Islamophobia in Asian countries. For instance, the Chinese government is alleged of incarcerating an estimated one million Uighur Muslims (ChinaFile Conversation, 2019). Since April 2017, at least ten Muslims have been lynched or killed in public in suspected hate crimes amid a rising tide of Islamophobia in India (Amnesty International, 2017). The foregoing reveals that Islamophobia is prevalent in most countries of the world, to the extent that some world leaders are driven by the fear of Islām.

As indicated in the work of Cipriani (2019), Islamophobia manifests itself across countries through physical or verbal attacks on property, places of worship and people, verbal or online threats of violence, vilification, and abuse, and policies or legislation that indirectly target or disproportionately affect Muslims, and unduly restrict their freedom of religion, discrimination in education, employment, housing, or access to goods and services. Other manifestations of this threat, includes ethnic and religious profiling and police abuse, and public pronouncements by some journalists and politicians that stigmatise Muslims as a group thereby disregarding their positive contributions to the communities and countries in which they live. The table below provides the selected cases of Islamophobic-related attacks and religious intolerance around the world between 2001 and 2019: 
Table 1. Selected Islamophobic-related cases and intolerance around the world, 2001-2019

\begin{tabular}{|c|c|c|c|c|}
\hline Date & Location & Modes of Attacks & Victims & Causes \\
\hline 30-04-01 & $\begin{array}{l}\text { Mawanella, } \\
\text { Central Province }\end{array}$ & $\begin{array}{l}\text { Shooting, melee } \\
\text { attack }\end{array}$ & $\begin{array}{c}2 \text { killed \& 15+ } \\
\text { injured; millions of } \\
\text { rupees destroyed }\end{array}$ & Racism; Xenophobia \\
\hline Oct. 01 & Dallas, Texas & Shooting & 1 killed & $\begin{array}{l}\text { Revenge on } 9 / 11 \\
\text { terrorist attacks }\end{array}$ \\
\hline 15-11-01 & Mesa, Arizona & Shooting & 1 killed & $\begin{array}{l}\text { Mistaken for an Arab } \\
\text { Muslim }\end{array}$ \\
\hline Feb. '03 & Qinghai & Mob attacks & $\begin{array}{l}100+\text { Hui Muslims } \\
\text { injured/Hui-owned } \\
\text { shops \& restaurants } \\
\text { destroyed }\end{array}$ & Misunderstanding \\
\hline $12-07-05$ & $\begin{array}{l}\text { Nottingham, } \\
\text { England }\end{array}$ & Melee attack & $\begin{array}{l}\text { A Muslim man } \\
\text { beaten to death }\end{array}$ & Anti-Muslim hatred \\
\hline 08-11-06 & Malegaon & Bombing & $\begin{array}{l}\text { Series of bombs } \\
\text { detonated in a } \\
\text { Muslim cemetery } \\
\text { (37 killed \& } 125+ \\
\text { injured) }\end{array}$ & $\begin{array}{l}\text { Extremism; Anti-Muslim } \\
\text { hatred }\end{array}$ \\
\hline $26-04-07$ & Kostroma & Melee attack & 2 injured & Racism; Xenophobia \\
\hline $30-06-07$ & Blackley, England & $\begin{array}{l}\text { Stabbing, } \\
\text { melee attack }\end{array}$ & $\begin{array}{l}\text { A Muslim religious } \\
\text { teacher stabbed }\end{array}$ & $\begin{array}{l}\text { Racism; Anti-Muslim } \\
\text { hatred }\end{array}$ \\
\hline $10-12-07$ & $\begin{array}{l}\text { Lauingen, } \\
\text { Bavaria }\end{array}$ & Bombing & A Mosque & Anti-Muslim hatred \\
\hline 01-01-08 & Sergiyev, Posad & Vandalism & $\begin{array}{c}\text { Muslim prayer } \\
\text { house destroyed }\end{array}$ & Racism; Xenophobia \\
\hline $05-02-08$ & Graz & Vandalism & $\begin{array}{c}\text { Close to } 60 \\
\text { tombstones of } \\
\text { Muslims desecrated }\end{array}$ & Extremism \\
\hline 07-02-08 & Liège & Melee attack & 2 women injured & Extremism/Hostility \\
\hline 09-02-08 & $\begin{array}{l}\text { Columbia, } \\
\text { Tennessee }\end{array}$ & Arson & $\begin{array}{c}\text { The Islamic Centre } \\
\text { in Columbia was } \\
\text { Firebombed }\end{array}$ & Extremism; Revenge \\
\hline $\begin{array}{l}\text { 10-24 } \\
\text { Mar'08 }\end{array}$ & Tibet & $\begin{array}{l}2008 \text { Tibetan } \\
\text { unrest }\end{array}$ & $\begin{array}{l}\text { Main Mosque in } \\
\text { Lasa burned down; } \\
18 \text { killed \& } 383 \\
\text { injured }\end{array}$ & Anti-Muslim hatred \\
\hline 06-04-08 & Arras & Vandalism & $\begin{array}{l}148 \text { Muslim graves } \\
\text { desecrated }\end{array}$ & Anti-Muslim hatred \\
\hline 20-04-08 & Toulouse & Arson & $\begin{array}{l}\text { Al-Salam Mosque } \\
\text { destroyed } / 1 \text { person } \\
\text { injured }\end{array}$ & Anti-Muslim hatred \\
\hline
\end{tabular}




\begin{tabular}{|c|c|c|c|c|}
\hline 02-06-08 & Copenhagen & Melee attack & $\begin{array}{l}\text { Spokesperson of IFS } \\
\text { injured }\end{array}$ & Racism \\
\hline June '08 & Milan & Bombing & $\begin{array}{l}\text { Two handmade } \\
\text { bombs were thrown } \\
\text { at the Islamic Centre }\end{array}$ & Anti-Muslim hatred \\
\hline $22-08-08$ & $\begin{array}{l}\text { Hastings, } \\
\text { England }\end{array}$ & Melee attack & 1 killed \& 2+ injured & $\begin{array}{c}\text { Racism; Anti-Muslim } \\
\text { hatred }\end{array}$ \\
\hline 03-11-08 & Lanao del Norte & Shooting & 5 killed by Ilaga & Anti-Muslim hatred \\
\hline 01-07-09 & Dresden, Saxony & Stabbing & 1 killed \& 1 injured & $\begin{array}{l}\text { Extremism; Anti-Muslim } \\
\text { hatred }\end{array}$ \\
\hline 13-12-09 & Castres & Vandalism & $\begin{array}{l}\text { Castres Mosque } \\
\text { vandalised }\end{array}$ & Anti-Muslim hatred \\
\hline $10-04-10$ & Karlovo & Arson & $\begin{array}{l}\text { A Mosque burned } \\
\text { down }\end{array}$ & Anti-Muslim hatred \\
\hline 03-05-10 & Nablus & Arson attack & $\begin{array}{l}\text { A Mosque in } \\
\text { the West Bank } \\
\text { destroyed }\end{array}$ & Xenophobia \\
\hline $10-05-10$ & $\begin{array}{l}\text { Babworth, } \\
\text { England }\end{array}$ & Bombing & $\begin{array}{l}\text { Attempt to kill } \\
\text { fellow Muslim } \\
\text { prisoners }\end{array}$ & $\begin{array}{l}\text { Terrorism; Anti-Muslim } \\
\text { hatred }\end{array}$ \\
\hline 09-06-10 & London, England & $\begin{array}{l}\text { Melee attack, } \\
\text { robbery }\end{array}$ & $\begin{array}{l}\text { A girl was burned } \\
\text { and robbed }\end{array}$ & Anti-Muslim hatred \\
\hline $10-06-10$ & Zlatoust & Vandalism & $\begin{array}{l}15 \text { tombstones of } \\
\text { Muslim cemetery } \\
\text { desecrated }\end{array}$ & Anti-Muslim hatred \\
\hline $23-08-10$ & Simferopol & Vandalism & $\begin{array}{l}\text { Seit-Settar Mosque } \\
\text { vandalised }\end{array}$ & Anti-Muslim hatred \\
\hline 24-01-11 & $\begin{array}{l}\text { Dearborn, } \\
\text { Michigan }\end{array}$ & $\begin{array}{l}\text { Attempted car } \\
\text { bombing }\end{array}$ & $\begin{array}{l}\text { Bombing plot of } \\
\text { Dearborn Mosque }\end{array}$ & Terrorism \\
\hline 20-03-11 & $\begin{array}{l}\text { Gainesville, } \\
\text { Florida }\end{array}$ & Burning & Qur'ān was burned & $\begin{array}{l}\text { Anti-Muslim hatred; } \\
\text { 9/11 Anniversary }\end{array}$ \\
\hline $22-07-11$ & Oslo \& Utøya & $\begin{array}{l}\text { Car bombing, } \\
\text { shooting }\end{array}$ & $\begin{array}{c}\text { Oslo (8 killed \& } \\
209 \text { injured; Utøya } \\
\text { (68 killed \& } 110 \\
\text { injured) }\end{array}$ & Islāmisation of Europe \\
\hline $22-11-11$ & $\begin{array}{l}\text { Mississauga, } \\
\text { Ontario }\end{array}$ & Melee attack & 1 woman injured & Anti-Muslim hatred \\
\hline $27-12-12$ & $\begin{array}{l}\text { New York City, } \\
\text { New York }\end{array}$ & Murder & $\begin{array}{l}\text { A Muslim Indian } \\
\text { man pushed onto } \\
\text { train track }\end{array}$ & Anti-Muslim hatred \\
\hline 29-04-13 & $\begin{array}{l}\text { Birmingham, } \\
\text { England }\end{array}$ & Stabbing & $\begin{array}{c}\text { Mohammed } \\
\text { Saleem was stabbed } \\
\text { to death while on } \\
\text { his way home from } \\
\text { Green Lane Masjid }\end{array}$ & Anti-Muslim hatred \\
\hline
\end{tabular}


ISLAMOPHOBIA AND RELIGIOUS INTOLERANCE: THREATS TO GLOBAL .....

\begin{tabular}{|c|c|c|c|c|}
\hline $19-11-13$ & Montreal, Quebec & Melee attack & 1 woman injured & Anti-Muslim hatred \\
\hline $31-12-13$ & $\begin{array}{l}\text { Vancouver, } \\
\text { British Columbia }\end{array}$ & Bomb threat & Vancouver Mosque & Anti-Muslim hatred \\
\hline $16-03-14$ & $\begin{array}{l}\text { Sacramento, } \\
\text { California }\end{array}$ & Shooting & $\begin{array}{l}\text { An Iraqi Muslim } \\
\text { killed }\end{array}$ & $\begin{array}{l}\text { Hatred for Middle } \\
\text { Eastern Muslims }\end{array}$ \\
\hline $29-03-14$ & Colchester, Essex & Stabbing & $\begin{array}{l}\text { A Saudi Arabian } \\
\text { student in hijab } \\
\text { killed }\end{array}$ & $\begin{array}{c}\text { Racism; Anti-Muslim } \\
\text { hatred }\end{array}$ \\
\hline 24-06-14 & Bambari & Shooting & 17 killed & Anti-Muslim hatred \\
\hline $02-07-14$ & Shuafat & $\begin{array}{l}\text { Kidnapping, melee } \\
\text { attack }\end{array}$ & 1 murdered & Xenophobia \\
\hline 24-11-14 & Lleida, Catalonia & Stabbing & $\begin{array}{l}5 \text { Arab Muslims } \\
\text { injured }\end{array}$ & $\begin{array}{c}\text { Racism; Anti-Muslim } \\
\text { hatred }\end{array}$ \\
\hline $26-11-14$ & Montreal, Quebec & Bomb threat & Montreal Mosque & Anti-Muslim hatred \\
\hline $04-12-14$ & $\begin{array}{l}\text { Kansas City, } \\
\text { Missouri }\end{array}$ & Vehicular attack & $\begin{array}{l}\text { A Muslim Somali- } \\
\text { American student } \\
\text { killed }\end{array}$ & Anti-Muslim hatred \\
\hline $14-01-15$ & Le Beaucet & Stabbing & 1 killed \& 1 injured & Terrorism; Extremism \\
\hline $28-09-15$ & Dadri & Melee attack & 1 killed \& 1 injured & Anti-Muslim hatred \\
\hline 03-11-15 & Kos & Melee attack & Several injured & Xenophobia \\
\hline $14-11-15$ & $\begin{array}{l}\text { Peterborough, } \\
\text { Ontario }\end{array}$ & Arson & $\begin{array}{c}\text { Peterborough } \\
\text { Mosque set on fire }\end{array}$ & Anti-Muslim hatred \\
\hline $26-12-15$ & Fresno, California & Melee attack & Sikh man killed & Mistaken for Muslim \\
\hline $27-02-16$ & $\begin{array}{l}\text { Enschede, } \\
\text { Overijssel }\end{array}$ & Arson & Mosque destroyed & Anti-Muslim hatred \\
\hline $23-03-16$ & Madrid & Arson & $\begin{array}{l}\text { The Great Mosque } \\
\text { of the M-30 of } \\
\text { Madrid damaged }\end{array}$ & Anti-Muslim hatred \\
\hline $23-06-16$ & Bago & Arson & Mosque demolished & Anti-Muslim hatred \\
\hline $29-06-16$ & $\begin{array}{l}\text { Minneapolis } \\
\text { Minnesota }\end{array}$ & Shooting & $\begin{array}{l}5 \text { Somali-Americans } \\
\text { shot \& } 2 \text { injured }\end{array}$ & Anti-Muslim hatred \\
\hline 02-07-16 & Hpakant & Arson & $\begin{array}{l}\text { Mosque burned } \\
\text { down }\end{array}$ & Anti-Muslim hatred \\
\hline 03-07-16 & $\begin{array}{l}\text { Sunderland, } \\
\text { England }\end{array}$ & Melee attack & $\begin{array}{l}\text { A Muslim woman } \\
\text { assaulted \& injured }\end{array}$ & $\begin{array}{c}\text { Racism; Anti-Muslim } \\
\text { hatred }\end{array}$ \\
\hline 07-08-16 & Tirana & Melee attack & $\begin{array}{c}1 \text { hijab woman } \\
\text { injured }\end{array}$ & Stigmatisation \\
\hline $13-08-16$ & $\begin{array}{l}\text { New York City, } \\
\text { New York }\end{array}$ & Shooting & $\begin{array}{l}2 \text { Muslim clerics } \\
\text { killed }\end{array}$ & Anti-Muslim hatred \\
\hline
\end{tabular}




\begin{tabular}{|c|c|c|c|c|}
\hline $11-10-16$ & $\begin{array}{l}\text { Garden City, } \\
\text { Kansas }\end{array}$ & $\begin{array}{l}\text { Attempted car } \\
\text { bombing }\end{array}$ & $\begin{array}{l}\text { Plan to detonate } \\
\text { explosives in } 4 \\
\text { trucks to bomb } \\
\text { Muslim refugees } \\
\text { apartment blocks }\end{array}$ & Anti-Muslim hatred \\
\hline $20-10-16$ & $\begin{array}{l}\text { Fort Smith, } \\
\text { Arkansas }\end{array}$ & Vandalism & 2 Mosques damaged & $\begin{array}{l}\text { Xenophobia; Anti-Muslim } \\
\text { hatred }\end{array}$ \\
\hline $07-11-16$ & $\begin{array}{l}\text { Barcelona, } \\
\text { Catalonia }\end{array}$ & Melee attack & $\begin{array}{l}\text { A pregnant Muslim } \\
\text { woman with a } \\
\text { Niqab injured }\end{array}$ & $\begin{array}{l}\text { Racism; Anti-Muslim } \\
\text { hatred }\end{array}$ \\
\hline $27-11-16$ & Sept-Îles, Quebec & Vandalism & $\begin{array}{l}\text { Sept-Îles Mosque } \\
\text { vandalised }\end{array}$ & Anti-Muslim hatred \\
\hline $27-11-16$ & Dresden, Saxony & Bombing & $\begin{array}{c}\text { A Mosque \& } \\
\text { convention centre } \\
\text { bombed }\end{array}$ & Xenophobia; Nationalism \\
\hline $06-12-16$ & Perth & Melee attack & $\begin{array}{l}1 \text { Muslim woman } \\
\text { injured }\end{array}$ & $\begin{array}{l}\text { Refusal to reply Xmas } \\
\text { greeting }\end{array}$ \\
\hline $10-12-16$ & $\begin{array}{l}\text { Simi Valley, } \\
\text { California }\end{array}$ & Stabbing & $\begin{array}{l}\text { A Muslim was } \\
\text { stabbed }\end{array}$ & Anti-Muslim hatred \\
\hline $16-12-16$ & London, England & Melee attack & $\begin{array}{l}\text { A Muslim woman } \\
\text { dragged along the } \\
\text { pavement by her } \\
\text { hijab \& injured }\end{array}$ & $\begin{array}{l}\text { Racism; Anti-Muslim } \\
\text { hatred }\end{array}$ \\
\hline $19-12-16$ & Zürich & Shooting & 2 killed \& 3 injured & $\begin{array}{l}\text { Racism; Anti-Muslim } \\
\text { hatred }\end{array}$ \\
\hline $28-12-16$ & $\begin{array}{l}\text { Colemborg, } \\
\text { Utrecht }\end{array}$ & Arson & $\begin{array}{l}\text { Islamic centre } \\
\text { burned }\end{array}$ & Anti-Muslim hatred \\
\hline $29-01-17$ & $\begin{array}{l}\text { Quebec City, } \\
\text { Quebec }\end{array}$ & Mass shooting & 6 killed \& 19 injured & Terrorism/Hate crime \\
\hline $9-02-17$ & Melbourne & Verbal attack & Group of Muslims & Auctioned Cartoons \\
\hline $21-02-17$ & Gothenburg & Arson & $\begin{array}{l}\text { An Islamic Centre } \\
\text { set on fire }\end{array}$ & Anti-Muslim hatred \\
\hline $23-02-17$ & Olathe, Kansas & Shooting & 1 killed \& 2 injured & $\begin{array}{l}\text { Racism; Anti-Muslim } \\
\text { hatred }\end{array}$ \\
\hline $10-03-17$ & Salem, Oregon & Melee attack & $\begin{array}{l}\text { Arab employee } \\
\text { injured }\end{array}$ & $\begin{array}{l}\text { Racism; Anti-Muslim } \\
\text { hatred }\end{array}$ \\
\hline $19-03-17$ & Melbourne & Stabbing & A priest injured & Mistaken for Muslim \\
\hline $11-05-17$ & Sydney & Melee attack & $\begin{array}{l}\text { 4 Muslim women } \\
\text { injured }\end{array}$ & Hate crime \\
\hline $26-05-17$ & Portland, Oregon & $\begin{array}{l}\text { Melee attack, } \\
\text { stabbing }\end{array}$ & 2 killed \& 1 injured & $\begin{array}{l}\text { Racism; Anti-Muslim } \\
\text { hatred }\end{array}$ \\
\hline $19-06-17$ & London, England & Vehicular attack & 1 killed \& 11 injured & $\begin{array}{l}\text { Terrorism; Anti-Muslim } \\
\text { hatred }\end{array}$ \\
\hline $24-06-17$ & Haryana & Melee attack & 1 killed \& 3 injured & Anti-Muslim hatred \\
\hline $4-07-17$ & Sittwe & Mob attacks & 1 killed \& 6 injured & 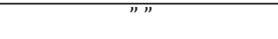 \\
\hline $12-07-17$ & Farrukhabad & $\begin{array}{l}\text { Melee attack, } \\
\text { robbery }\end{array}$ & 10 injured & $\begin{array}{l}\text { Anti-Muslim hatred; } \\
\text { Racism }\end{array}$ \\
\hline
\end{tabular}




\begin{tabular}{|c|c|c|c|c|}
\hline 03-08-17 & $\begin{array}{l}\text { Brazil Rio de } \\
\text { Janeiro }\end{array}$ & Melee attack & $\begin{array}{l}\text { A Muslim food-cart } \\
\text { owner injured }\end{array}$ & Xenophobia; Hate crime \\
\hline $05-08-17$ & $\begin{array}{l}\text { Bloomington, } \\
\text { Minnesota }\end{array}$ & Bombing & $\begin{array}{c}\text { Dar Al-Farooq } \\
\text { Islamic Centre } \\
\text { bombed }\end{array}$ & Anti-Muslim hatred \\
\hline 23-08-17 & Madrid & Melee attack & $\begin{array}{l}\text { A Muslim woman } \\
\text { injured }\end{array}$ & $\begin{array}{l}\text { Racism; Anti-Muslim } \\
\text { hatred }\end{array}$ \\
\hline $13-09-17$ & $\begin{array}{l}\text { New York City, } \\
\text { New York }\end{array}$ & Melee attack & $\begin{array}{c}1 \text { Jewish woman \& } \\
\text { her daughter beaten } \\
\text { up }\end{array}$ & Mistaken for Muslims \\
\hline $11-10-17$ & Kembe & Shooting & 25 killed & Anti-Muslim hatred \\
\hline $20-11-17$ & $\begin{array}{l}\text { Leicester, } \\
\text { England }\end{array}$ & Vehicular attack & $\begin{array}{l}\text { A Muslim woman } \\
\text { injured }\end{array}$ & Racism \\
\hline 15-03-19 & Christchurch & Shooting & $\begin{array}{l}51 \text { killed \& 50+ } \\
\text { injured }\end{array}$ & $\begin{array}{l}\text { Extremism; Xenophobia; } \\
\text { Anti-Muslim hatred }\end{array}$ \\
\hline Mar'19 & Colindale & Gun gesture & $\begin{array}{l}\text { A Muslim mother \& } \\
\text { her daughter }\end{array}$ & $\begin{array}{l}\text { Anti-Muslim hatred } \\
\text { linked to NZ attacks }\end{array}$ \\
\hline Mar'19 & Oxford & Harassment & $\begin{array}{l}\text { A Muslim woman } \\
\text { with hijab }\end{array}$ & Anti-Muslim hatred \\
\hline Mar'19 & Scotland & Melee attack & Mosque attacked & Anti-Muslim hatred \\
\hline
\end{tabular}

Source: Compiled by the Author from Wikipedia Encyclopedia and other websites.

The list of selected cases of Islamophobic incidents around the world, as presented in Table 1 reveals that there is no continent spared of Islamophobic threats. The European countries maintain the highest records of this malady, followed by the American and Asian countries. AntiMuslim hatred constitutes the major cause of the recorded incidents followed by racism and xenophobia. Others include extremism, hate crime and revenge. Going by the records, the White supremacists, and Right-wing, Far-right, and Neo-Nazi extremists committed a considerable number of these attacks on Muslims. It also shows that Muslim women, especially those who display a visible manifestation of their religious identity, such as women wearing Hijāb or Niqā b, have been the 
worse hit of the attacks. Men with beard and or with religious attires, such as Turban, have also been the target.

It is quite revealing that most melee attacks on Muslims and vandalism of Mosques and Tombstones of dead Muslims can be traced to anti-Muslim hatred cum religious intolerance orchestrated by non-Muslims, the phenomenon which reveals hate speech, hate crime, as well as discrimination directed against group of Muslims or individual Muslim. Xenophobia is another cause of Islamophobia. Xenophobia connotes the fear and hatred of foreigners. It is perpetuated through a dynamic public rhetoric by indigenes that actively stigmatises and vilifies migrants by playing up the 'threat' posed by their presence and making them scapegoats for socio-economic woes in their host country (Crush, 2015). While xenophobia indicates dislike of/or prejudice against foreigners, Islamophobia reflects hatred, hostility or prejudice against Islām/Muslims, either such person hail from same country or other countries. As Alan (2011) has posited, Islamophobia is nothing more than xenophobia or racism wrapped in religious terms. As a matter of fact, an individual or group of people may suffer both xenophobic and Islamophobic threats or attacks. For instance, a Muslim from African or Arab countries working in Europe may be subjected to both hate crimes.

Likewise, there is a growing trend of Far-right movement or Right-wing extremists which has doubled in number in the recent years. A report says Islamophobia has become the driving force behind the rise of Far-right movements in the UK hence anti-Muslim prejudice has replaced immigration as the "key driver" of the far-right groups (BBC, 18 February 2019). The State of Hate report (2019: 3) indicates that the U.K. is 
witnessing a growing threat of Far-right terrorism, while the extreme Far-right is getting more extreme thereby tapping into the political rage and discontents that are prevalent in society. This report blames the 2017 terror attacks in London and Manchester for a "lasting negative impact" on attitudes toward Muslims. Similar report established that there are approximately 100 White supremacist groups operating in Canada, stating that the presence of White supremacist nationalism gaining renewed impetus across Canada over the past two years. The Canada Border Services Agency also found that "Right-wing ideology" in Alberta is growing (TheConversation, 6 February 2019). Consequently, members of the White supremacist groups have contributed to the breeding ground of Islamophobic fear and hate crimes not only in Canada but other western countries.

The foregoing shows that Islamophobia has been aggravated by high-profile terrorist attacks carried out by extremists at both end - Muslim extremists and Right-wing extremists. No doubt, the 9/11 terrorist attacks drastically changed public opinions of Muslims. Since then, terrorist acts, such as the attacks by violent Jihadists in London, Paris, Brussels, and Barcelona, have increased fear and anxiety. The use of Islām by extremists to justify their terrorist acts had instigated many westerners and even Africans to perceive Islām as a threat and fear Muslims as the enemy (Cipriani, 2019).

Corroborating Cipriani's argument, Abubakar (2019) attributes the major cause of Islamophobia in the world to the spate of terrorist attacks perpetrated by extremists who hide under the name of Islām. He observed further that "due to wrong religious ideology, some sycophants twist the religious 
texts to suit their agenda with the major aim at causing mayhem, chaos and instability in a hitherto peaceful society." Hence, series of attacks carried out by ISIS, DAESH, al-Shabāb, Boko Harām, and other religious motivated insurgent groups related to Islām sparked hate crimes and Islamophobic attacks against innocent Muslims in Europe, including the Christchurch Mosque attacks in New Zealand, where over 50 worshippers were killed by a White supremacist terrorist (Abubakar, 2019).

Conversely, a 2017 report has revealed that most of the designated terrorist groups in U.S. in the recent past are right-wing extremists and not Muslims. A joint project by the Investigative Fund at the Nation Institute, Centre for Investigative Reporting (CIR) examined 201 designated terrorism incidents within the U.S. from 2008 and 2016. The report reveals that "Right-wing extremist were behind nearly twice as many incidents" as terror acts associated with those identified as "Islāmist-domestic terrorism". Right-wing extremists, often White supremacists, were responsible for 115 incidents within the same period (Sampathkumar, 2017). Other inducing causes include Orientalists' perceptions of Islām, poor knowledge about Islām, growing number of Islamophobic industry, fear of domination, hate speeches of public figures, and public anxiety over immigrants and immigration programmes.

\section{Nexus and implications of Islamophobia and religious intolerance for global peace and harmonious co-existence}

There has been a growth in organisations that portray Islām as a threat to the world's peace. Consequently, 
Islamophobic incidents and religious intolerance have steadily increased over the past few years across the continent. The former Special Rapporteur appointed by the United Nations Commission on Human Rights, Angelo d'Almeida Ribeiro, once stated that "discrimination based upon religion or conviction exists in almost all economic, social, and ideological systems and in all parts of the world" (Religious Intolerance Today, 1999:3-4). Against the backdrop of the United Nations' Universal Declaration of Human Rights (UNUDHR) (1948) which upholds the right to free expression of religious belief in Articles 18 and 19 wherein Article 2 forbids discrimination on the basis of religion, the Human Rights Council in its Sixteenth session (2011) adopted Resolution 16/18 on "Combating intolerance, negative stereotyping and stigmatisation of, and discrimination, incitement to violence and violence against, persons based on religion or belief." This resolution was hailed by stakeholders from all regions and faiths as a turning point in international efforts to confronting religious intolerance. The question of whether this resolution is effectively utilised to curb religious intolerance in the world could be glaringly adjudged by all and sundry.

Although, intolerance against and persecution of Muslims is not new. In the earliest period of Islām, Muslims in Makkah were subjected to all forms of abuse, torture, harassment and discrimination by the pagans, who felt threatened by the fast-growing Abrahamic religion introduced by Prophet Muhammad [Peace and Blessings of Allāh be Upon Him]. The noble Prophet [PBUH] himself and considerable number of new converts, such as Sumayyah bint Khabbāb and her husband, Yasir ibn Amir were tortured 
and martyred for having adopted the faith of Islām; Sa'd ibn 'Ubādah was tortured and almost killed; daughters of the Prophet were divorced, and two sons - Hārith and 'Abdullāh - of same father, Yasir, were tortured to death. Moreover, Bilāl ibn Rabah, Ammar ibn Yāsir, Abū Fakīha, Hārithah bint alMu'ammil, Zunayra al-Rumiya bint al-Mu'ammil, Ghaziyyah bint Jābir, Sumayya bint Khayyāt, and a host of others, were made to face random persecutions due to their proclamation of Islamic faith.

After over fourteen centuries, Muslim Ummah (population) have continued to be the target for persecution by non-Muslims. Today, Muslims remain targets of aggression and discrimination in many countries of the world. Adherents of Muslim faith have been jailed, deported, and/or killed in several attacks by non-Muslim extremists and terrorists. Over the past years, reports from international and civil society organisations have pointed to an increase in insecurity among Muslims, persistent intolerance and racism, as well as form of discrimination directed against individuals women in particular - with a Muslim background, and Muslim communities in the European countries (European Commission, 2019).

Thus, the paper proceeds by arguing that Islamophobia is a consequent effect of religious intolerance. This is because arising from intolerance is the fear of or prejudiced viewpoint towards Islām, Muslims and matters pertaining to them. Religious intolerance is broader in scope, while Islamophobia is narrower. In other words, Islamophobia is subsumed under religious intolerance. Both are characterised by discrimination and hatred towards one set of people due 
to their faith. Generally, religious intolerance does not only relate to unpleasant attitude toward Islām but other religions, whereas Islamophobia is specifically meant to promote hatred, fear and discrimination against Muslims. This agrees with Roald's (2004) perception that Islamophobia should be recognised as a form of intolerance alongside xenophobia and anti-Semitism.

Corroborating the above argument, Walzer (2015) contends that Islamophobia is a form of religious intolerance and hatred. He asserts further that contemporary Muslims in Europe and United States, are deliberately misunderstood and misrepresented; Muslim immigrants in western countries are regarded as potential terrorists, while failing to acknowledge the towering achievements of Muslim philosophers, poets, and artists over many centuries. Other causes of religious intolerance as emphasised by Fan, Jerry and Nnaraonye (2014), include religious bigotry, indoctrination, factionalism within a religious group, power tussles, ignorance and misunderstanding of the central teaching and practices of the different religions, as well as politicisation of religious matter.

However, Islamophobia and religious intolerance are a threat to human life and property. They are an affront to fundamental human rights and dignity of Muslims, as well as a threat to freedom to practice their faith and spiritual uplift. President Jimmy Carter in his foreword on Countering the Islamophobia Industry: toward More Effective Strategies lamented on the unprecedented rise of anti-Muslim hatred crimes where Muslims are subjected to discriminatory and unconstitutional practices. Carter believes that such actions do not only infringe on the freedom of Muslims to practice 
their faith, but also marginalise them as engaged citizens (Carter, 2018:4).

Islamophobic attacks had continued to paint a gloomy picture about the social cohesion and mutual collaboration between Muslims and non-Muslims around the world. Furthermore, attacks on Muslims had contributed to a growing sense of insecurity among many Muslims and intensified religious tensions. The threats had increasingly compounded fears, hostilities and intimidations of the followers of Islamic faith. The fears of discrimination and constant dehumanisation had resulted in anxiety and depression for Muslims and those perceived to be one. Similarly, veiled Muslim women and bearded Muslim men that were victims of hate crimes had been subjected to unfortunate feelings of humiliation, anger, sadness, isolation and disgust (Malik, 2016).

In recent years, there have been proliferations of laws passed in the United States that prey on innocent Muslim men and women. Itaoui and Elsheikh (2018) identified 216 bills introduced in 43 State legislatures in the U.S. since 2010 that sought to ban "Sharīah law," or a set of guiding principles, from being considered in the U.S. courts. These anti-Sharīah laws had exacerbated already-hostile climate of fear of Muslims, and further institutionalised and legalised their exclusion from society (Kopf \& Merelli, 2017). The passage of policies or legislation has indirectly targeted or disproportionately affected Muslims, and unduly restricted their freedom of religion, such as bans on wearing visible religious and cultural symbols, laws against facial concealment, and/or bans on building Mosques with minarets (Cipriani, 2019). 
Ostensibly, promoting religious intolerance is a threat against tolerance, trust and peaceful co-existence among Muslims and non-Muslims. Peaceful co-existence presupposes an obligation to live together in peace rather than in constant hostility. Intolerance among different religious adherents which has become veracious and alarming has had obvious effect on peaceful co-existence. What more, both Islamophobia and religious intolerance have led to increasing division along religious line and identity crisis in the society.

\section{Resolutions towards curbing rising trend of Islamophobia and religious intolerance}

No doubt, the barbaric attacks against Muslims sponsored by state officials, far-right public figures and political leaders, and motivated by anti-Islamic body language of government and political leaders, anti-Muslim hatred, fear of domination, anti-Muslim rhetoric of mainstream media, extremism, and misunderstanding of Islām and its tenets, etc and perpetrated by individual extremists, who detest Islām and its adherents, are worrisome and dangerous. The pattern of stereotypes committed against Muslims with seemingly impunity, is deeply worrying. Unfortunately, the world governments and their leaders have done little to show their disproval of this violence. Therefore, to effectively curb the rising trend of Islamophobia and religious intolerance, as well as ameliorating the current threats facing Muslims across the world, the following resolutions are proffered:

First, global inter-faith dialogue needs to be urgently considered, where lingering issues pertaining to 'religion and the crises associated with it' would be extensively discussed 
and drastically sorted out. The international community should devise possible means of curbing growing intolerance, fear and hatred against Muslims, perhaps through peaceful deliberation among world leaders. The United Nations (UN) established with the sole aims of guaranteeing peace, security, mutual co-operation and tolerance, among world nations, should wake to its responsibilities. Thus, the UN and its Human Rights Council need to be strengthened. The Human Rights Council's Resolution 16/18 on “Combating intolerance, negative stereotyping and stigmatisation of, and discrimination, incitement to violence and violence against, persons based on religion or belief" must be re-energised and member-states be enjoined to respond positively towards its implementation in their respective states.

Islām has oftentimes been misrepresented in the mainstream media. Thus, media stereotyping of Muslims and perpetuation of prejudice and biased information about Islām and Muslim communities, has further fuelled rise in Islamophobic attacks (Benn \& Jawad, 2003; Institute for Social Policy and Understanding [ISPU], 2018). Pratt (2016) stresses that a false understanding of Islām is shaped by long-standing bias and fuelled by contemporary media representation. Thus, the media industries should be called to order to refrain from normalising Islamophobia, promoting religious intolerance, and spreading negative narratives against Islām and Muslims. Conflating Islām with violent extremism and terrorism as if everything about the religion and its adherents is violence and terror, should be out rightly discouraged. Muslim rights groups and elites should also invest in the media to help change damning anti-Islām narratives. 
Further, there is the need for outright condemnation of Islamophobia and religious intolerance. This paper also shares the view of European Commission against Racism and Intolerance (2000) that "Governments have duties to uphold the freedom of religion and guarantee that the followers of all faiths may live and thrive in their societies without religious intolerance and discrimination." This further implies that the rights of Muslim to practise their religion should be enabled and protected in all spheres of public life. Perhaps if the existing resolutions, strategies, approaches, recommendations and policies were effectively coined and implemented, the world might have been free from chaos and crises occasioned by religious intolerance and discrimination. Efforts should be intensified by world religious and intergovernmental bodies, such as the United Nations, the European Commission, etcetera, to absolutely condemn Islamophobia and attacks on Muslims by tagging Islamophobic-related incident - a crime against humanity, while imposing appropriate sanctions on any defaulting country in case of violation of such rules.

Curbing of hate speeches by public figures and world leaders is non-negotiable. Hate speeches by public figures have absolutely inspired Far-right nationalists, right-wing extremists, White supremacists and other Islamophobes to perpetrate evil attacks against Muslims. When public figures use their platforms for hate speech, they become responsible for normalising feelings of bigotry thatcan lead to hate crimes againstinnocentindividuals (Malik, 2016). Hate speech encourages disunity which is inimical to our collective survival and wellbeing. Therefore, public figures and world leaders should be sanctioned to desist from official or non-official statements that can fuel hate crimes, ember of 
violence, and by extension, endanger the lives of individual and or group of Muslims.

Another resolution is the security of Mosque, Islamic centres and cemeteries. The Mosque management should devise effective security measures within the ambits of the law to protect places of worship, Islamic centres, worshippers and their properties, against any attack from bandits and extremists. Also, the governments must secure members of Muslim communities from intolerance and discrimination by providing necessary and adequate security assistance to the Mosques and worshippers in a bid to protect lives and maintain law and order in the society. Protection of places of worship, religious sites and cemeteries, and to take measures in cases where they are vulnerable to vandalism or destruction, is a significant part of the Resolution 16/18.

In addition to security measure, religious leaders and scholars have a critical role to play. Religious leaders must preach peace, tolerance and love, rather than fuelling religious bigotry, hatred and extremism through their radical teachings. In furtherance to this, scholars of Abrahamic religion must be guided by the notions of love, unity and peaceful coexistence in their writings and public discourses. Seeking for true knowledge about other religions is encouraged rather than following blind obedience. This is why Al-Qaradāwī (1987) warns on the dangers of blind obedience, bigotry and intolerance, harshness in the treatment of people, roughness in the manner of approach, and rudeness in calling people to Islām, all which he says, are contrary to the teachings of the holy Qur'ān and traditions of the noble Prophet [PBUH]. 
More importantly, is the urgent need for attitudinal change among religious leaders and followers. Muslims must represent Islām, according to the dictates of the Sharīah and avoid involvement in any form of extremism, revenge, but embrace peace in all ramifications. The verses of the holy Qur'ān unequivocally warn Muslims of extremism or fanaticism (see Qur'ān 2:190, 2:256, 5:77, 8:61, 11:112 \& $41: 34)$. On the other hand, the followers of other faith should also abide by the tenets and contents of their scriptures. No messenger or Prophet of God was sent to inculcate in his disciples cum devotees spread of violence, intolerance and hatred. It is suggested therefore that religious leaders and followers should encourage tolerance, love, and sue for peace within and among the people of same or different religions.

\section{Conclusion}

Muslims across the world had been subjected to physical and verbal attacks, and directly or indirectly made the target of discrimination, hate speeches, stigmatisation, namecalling, abuse, vilification, psychological trauma, negative stereotypes, harassment and hostility. These, as established in this research, are caused by anti-Muslim hatred-cum-religious intolerance, racism, xenophobia, growing trend of far-right or right-wing extremist movements, and high-profile terrorist attacks. In the process, many had lost their lives, members of their family, relatives and loved ones, as well as their properties, to melee or vehicular attacks, shooting, stabbing, kidnapping, arson, vandalism and bombing. Besides, Mosques, Islamic centres, Islām's sacred symbols and scripture, as well as Muslim cemeteries, are not spared. 
Equally, the spread of fear, discrimination and intolerance championed by non-Muslims against Islām and its adherents had threatened fundamental human rights and dignity of Muslims populace, as well as mutual collaboration, trust, diversity, harmonious and peaceful co-existence, the world over. The incessant attacks had also culminated in rising fear of insecurity, and distrust between Muslims and non-Muslims. Hence, concerted efforts should be made by stakeholders towards curbing rising trends of Islamophobia and religious intolerance in contemporary world, following the suggested resolutions.

\section{REFERENCES}

Abadi, H. (2018). The Carter Centre works to understand and counter the rise of Islamophobia. In Countering the Islamophobia industry toward more effective strategies: symposium report and analysis (pp. 5-7). Atlanta, GA: The Carter Centre.

Abdulhadi, R. I. (2018). The Islamophobia and Israel lobby industries: overlapping interconnection and antiracist policy recommendations. In Countering the Islamophobia industry toward more effective strategies: symposium report and analysis (pp. 14-22). Atlanta, GA: The Carter Centre.

Abubakar, R. (2019, June 22). Terrorism, media stereotypes fuel Islamophobia - expert. Muslim News. Retrieved from https://muslimnews.com.ng/2019/06/22/terrorismmedia-stereotypes-fuel- Islamophobia-expert/

Abu Sway, M. (2005). Islamophobia: meaning, manifestations, causes. Palestine-Israel Journal (Anti-Semitism \& 
Islamophobia), 12(2). Retrieved from https://pij.org/ articles/342/Islamophobia-meaning-manifestationscauses

Alan, J. (2011, March 6). The idea of 'Islamophobia'. World Affairs. Retrieved from http://www.worldaffairsjournal. org/blog/alan-johnson/idea-Islamophobia

Al-Qaradawi, Y. (1987). Islamic awakening between rejection and extremism. International Institute of Islamic Thought.

American Civil Liberties Union [ACLU]. (2019). Nationwide anti-Mosque activities. Retrieved from https://www. aclu.org/issues/national-security/discriminatoryprofiling/nationwide-anti-mosque-activity

Amnesty International. (2017, June 28). In India: hate crimes against Muslims and rising Islamophobia must be condemned. Retrieved from https://www.amnesty.nl/ actueel/india-hate-crimes-against-muslims-and-risingIslamophobia-must-be-condemned

Benn, T., \& Jawad, H. A. (2003). Muslim women in the United Kingdom and beyond: experiences and images. Leiden, Boston: Brill.

Bleich, E. (2011). What is Islamophobia and how much is there? theorising and measuring an emerging comparative concept. American Behavioural Scientist, 55(12), 15811600.

Chalkley-Rhoden, S. (2014, October 29). One in four Australians has negative attitude towards Muslims: report. $A B C$ News. Retrieved from https://www.abc.net. au/news/2014-10-29/one-in-four-australians-had-anegative-attitude-towards-muslims/5849744 
Cipriani, L. (2019). Islamophobia in Europe. Retrieved from https://www.opensocietyfoundations.org/explainers/ Islamophobia-europe

Crush,J.(2015).Globalmigration. In InternationalEncyclopedia of the Social \& Behavioural Sciences (Second Edition) (pp. 169-173). doi:https://doi.org/10.1016/B978-0-08097086-8.72113-1

Encyclopedia of World Problems and Human Potential. (2017). Religious intolerance. Retrieved from http:// encyclopedia.uia.org/en/problem/139538

Esposito, J. L. (2017). Foreword to the first edition. In N. C. Lean, \& J. G. Shaheen (Eds.), The Islamophobia industry: how the right manufactures hatred of Muslims (pp. xivxviii). London: Pluto Press.

European Commission Against Racism and Intolerance [ECRI]. (2000). General policy recommendation no. 5: combating intolerance and discrimination against Muslims. Strasbourg: Council of Europe.

European Commission. (2019, June 18). Combating antiMuslim hatred. Retrieved from https://ec.europa.eu/ newsroom/just/item-detail.cfm?item id=50085

Frost, D. (2008). Islamophobia: examining causal links between the media and "race hate" from "below". International Journal of Sociology and Social Policy, 28(11/12), 564-578.

Ghani, N. (2019). The rise of Islamophobia in the United States: patterns, perpetrators and reactions. American International Journal of Contemporary Research, 8(4), 1-12.

Graham-McLay, C. (2019, May 2). Death toll in New Zealand Mosque shootings rises to 51. The New York Times. 
Retrieved from https://www.nytimes.com/2019/ 05/02/world/asia/new-zealand-attack-death-toll.html

Human Rights Council. (2011). 16/18 combating intolerance, negative stereotyping and stigmatisation of, and discrimination, incitement to violence and violence against, persons based on religion or belief, Sixteenth session, 24 March. United Nations: HRC.

Hussain, Y., \& Bagguley, P. (2012). Securitised citizens: Islamophobia, racism and the 7/7 London bombings. The Sociological Review, 60(4), 715-734.

Institute for Social Policy and Understanding [ISPU]. (2018, April 30). American Muslim poll 2018: full report. Retrieved from https://www.ispu.org/americanmuslim-poll-2018-full-report

International Civil Liberties Alliance [ICLA]. (2013). Islamophobia definition. Retrieved from http:// libertiesalliance.org/wp-content/upload/2013/09/ Islamophobia-definition.pdf

Islamophobia and hate crimes continue to rise in Canada. (2019, February 6). TheConversation. Retrieved from https://theconversation.com/Islamophobia-and-hatecrimes-continue-to-rise-in-canada-110635

Islamophobia in China. (2019, May 14). ChinaFile Conversation. Retrieved from http://www.chinafile. com/conversation/Islamophobia-china

Islamophobia and understanding anti-muslim sentiment in the West. (n.d.). Gallup News. Retrieved from https:// news.gallup.com/poll/157082/Islamophobiaunderstanding-anti-muslim-sentiment-west.aspx 
Istriyani, R., \& Yuliatun. (2016). Media: causes and strategies to overcome Islamophobia (psychological and sociological study). QIJIS: Qudus International Journal of Islamic Studies, 4(2), 201-217.

Itaoui, R., \& Elsheikh, E. (2018). Islamophobia in the United States: a reading resource pack. Berkeley, CA: Haas Institute for a Fair and Inclusive Society, University of California.

It's anti-Muslim hatred, not 'Islamophobia'. (2019, May 16). TRTWorld Magazine. Retrieved from https://www. trtworld.com/magazine/it-s-anti-muslim-hatred-notIslamophobia-26698

Kareem, M. A. (2014). Introduction to world religions. Lagos: National Open University of Nigeria.

Kopf, D., \& Merelli, A. (2017, September 14). The legalisation of Islamophobia is underway in the United States. Retrieved from https://qz.com/1074415/anti-shariabills-exploit-Islamophobia-in-the-us-like-anti-catholicpolitics-used-to/

Kumar, D. (2018). Expanding the definition of Islamophobia: ideology, empire, and the war on terror. In Countering the Islamophobia industry toward more effective strategies: symposium report and analysis (pp. 61-64). Atlanta, GA: The Carter Centre.

Lee, S. A., Gibbons, J. A., Thompson, J. M., \& Timani, H. S. (2009). The Islamophobia scale: instrument development and initial validation. International Journal for the Psychology of Religion, 19(2), 92-105.

Limon, M., Ghanea, N., \& Power, H. (2014). Policy report: combating global religious intolerance. The 
implementation of Human Rights Council resolution 16/18. Retrieved from https://www.universal-rights. org/urg-policy-reports/-combatting-global-religiousintolerance-the-implementation-of-human-rightscouncil-resolution-1618/

Malik, S. (2016, March 25). When public figures normalise hate. Centre for American Progress. Retrieved from https://www.americanprogress.org/issues/race/ news/ 2016/03/25/134070/when-public-figuresnormalise-hate/

Mohamed Osman, M. N. (2017). Understanding Islamophobia in Asia: the cases of Myanmar and Malaysia. Islamophobia Studies Journal, 4(1), 17-36.

Nearly half of Australians are anti-Muslim: study. (2011, February 23). $A B C$ News. Retrieved from https://www.abc.net.au/ news/2011-02-23/nearly-half-of-australians-are-antimuslim- study/1954194

Osiewicz, P. (2017, September 19). Europe's Islamophobia and the refugee crisis. Retrieved from https://www.mei.edu/ publications/europes-Islamophobia-and-refugee-crisis

Pratt, D. (2016). Islam as feared other: perception and reaction. In D. Pratt, \& R. Woodlock (Eds.), Fear of Muslims? International perspectives on Islamophobia (pp. 31-43). New York: Springer International Publishing.

Raji, A., Araba-Yusuf, T. K., \& Ilemaiye, F. O. (2015). Religious tolerance as a means for peaceful co-existence in Nigeria. Retrieved from https://www.researchgate. net/publication/322930714/religious tolerance as a means for peaceful co-existence in nigeria 
Religious Intolerance Today Awake. (1999). Religious intolerance. Retrieved from https://wol.jw.org/en/ $\mathrm{wol} / \mathrm{d} / \mathrm{r} 1 / \mathrm{lp}-\mathrm{e} / 101999001 \# \mathrm{~h}=3$

Roald, A. S. (2004). New Muslims in the European context: the experience of Scandinavian converts. Leiden, Boston: Brill.

Runnymede Trust. (1997). Islamophobia: a challenge for us all. London: The Runnymede Trust.

Runnymede Trust. (2017). Islamophobia: still a challenge for us all. London: London: The Runnymede Trust.

Sampathkumar, M. (2017, June 23). Majority of terrorists who have attacked America are not Muslim, new study finds. Independent. Retrieved from https://www. independent.co.uk/news/world/americas/us-politics/ terrorism-right-wing-america-muslims-Islām-whitesupremacists-study-a7805831.html

Todd, D. (2009, August 15). The numbers game: will Muslims dominate the west? Vancouver Sun. Retrieved from https://vancouversun.com/news/staff-blogs/thenumbers-game-will-muslims-dominate-the-west

Triandafyllidou, A. (2010). Muslims in 21 ${ }^{\text {st }}$ century Europe: structural and cultural perspectives. New York: Routledge.

Vertovec, S. (2002). Islamophobia and Muslim recognition in Britain. Retrieved from doi:10.1093/ acprof:oso/9780195148053.003.0002

Walzer, M. (2015). Islamism and the left. Dissent Magazine. Retrieved from https://www.dissentmagazine.org/ article/Islāmism-and-the-left

Zúquete, J. P. (2008). The European extreme-right and Islam: new directions? Journal of Political Ideologies, 13, 321-344. 